\title{
High prevalence of mupirocin-resistant staphylococci in a dialysis unit where mupirocin and chlorhexidine are routinely used for prevention of catheter-related infections
}

In our centre, $65 \%$ of newly diagnosed endstage renal disease (ESRD) patients start haemodialysis with a tunnelled central venous catheter (CVC) (Teo et al., 2010). The median lifespan of a CVC until transition to a permanent vascular access (arteriovenous fistula or graft) or to another treatment modality is 105 days (interquartile range: 69-193 days), while the median time to a CVC-related infection is 72 days (inter-quartile range: 21-151 days).

As part of a clinical process improvement project to reduce these infections, we implemented inpatient and outpatient antisepsis protocols from August 2008 comprising daily $2 \%$ chlorhexidine bodywash along with application of $2 \%$ mupirocin ointment to the anterior nares and CVC exit-sites of patients daily. These protocols are discontinued only upon CVC removal. Following these changes, the rate of infections did not change significantly from our historical (January 2007-July 2008) mean of 1-2 per 1000 catheter-days (Teo et al., 2011). Antisepsis protocols have been shown to reduce the risk of infections (Batra et al., 2010; Bleasdale et al., 2007; McCann \& Moore, 2010; Sheng et al., 2009). However, emergence of mupirocin resistance has been documented in peritoneal dialysis patients, but is less well studied in haemodialysis patients

(Tacconelli et al., 2003). Also, whether chlorhexidine resistance develops as a result of this practice is unclear (Batra et al., 2010). We performed this prospective surveillance study to determine the prevalence of mupirocin- and chlorhexidine-resistance genes in staphylococcal carriage following these practice changes.

After institutional review board approval, consenting general ward inpatients with

A supplementary figure showing PFGE patterns of mupirocin-resistant staphylococci from the dialysis unit patients is available with the online version of this paper.
ESRD dialysed via tunnelled CVC underwent anterior nares and catheter exit-site swabbing using sterile calcium alginate-tipped swabs upon each hospitalization between May and August 2009. Specimens were swabbed onto $5 \%$ sheep blood agar plates and in cooked meat broth, and incubated at $35{ }^{\circ} \mathrm{C}$ overnight. If no staphylococci were seen on the primary plate, this was reincubated for another $24 \mathrm{~h}$ and the cooked meat broth was subcultured onto a CNA (Columbia agar with $5 \%$ sheep blood containing colistin and nalidixic acid) plate. Staphylococci were identified by colony morphology, Gram stain and latex agglutination with Pastorex Staph Plus (Bio-Rad). Coagulase-negative staphylococci (CoNS) were not further speciated. Mupirocin MICs were determined via Etest (AB Biodisk), while the presence of mec and ileS-2 genes encoding meticillin and high-level mupirocin resistance, respectively, was detected using a multiplex PCR protocol (Pérez-Roth et al., 2001). We also tested for the presence of $s m r$ and $q a c A / q a c B$ efflux genes, which are associated with reduced susceptibility to chlorhexidine (Noguchi et al., 2005). Isolates were typed using PFGE (Maslow et al., 1993).

Thirty-two patients were recruited, of whom nine patients were on their first hospitalization and haemodialysis. There were altogether 66 hospitalizations (minimum 1, maximum 5; median 1). All swabs were culture-positive, yielding 140 distinct Staphylococcus species isolates. These comprised 86 meticillin-resistant CoNS (MR-CoNS), 36 meticillinsusceptible CoNS (MS-CoNS), 11 meticillin-resistant Staphylococcus aureus (MRSA) and 7 meticillin-susceptible S. aureus (MSSA).

The distribution of organisms and antiseptic-resistance genes according to mupirocin MIC is shown in Table 1. Staphylococcal isolates positive for ileS-2 and high-level mupirocin resistance were isolated from $23(71.9 \%)$ previously hospitalized patients on initial swabs, but none were isolated from the remaining nine patients during their first hospitalization. However, three of these patients had acquired ileS-2-positive MR-CoNS by their second hospitalization, replacing initially mupirocin-susceptible MS-CoNS. Three (3/ 5) MRSA-positive patients were colonized with mupirocin-resistant MRSA, one of whom had concurrent mupirocin-resistant MR-CoNS isolated. No S. aureus isolate was found to have low-level mupirocin resistance. Mupirocin-resistant CoNS were more likely to be concomitantly meticillin resistant (chi-square; $P<0.01$ ). The majority of staphylococcal isolates were positive for qacA/B (30 patients; $93.8 \%$ ) but $s m r$ prevalence was lower (18 patients; $56.3 \%)$. One patient was colonized with mupirocin-susceptible MS-CoNS for all hospitalizations. Five patients were not readmitted.

PFGE results did not demonstrate any predominant CoNS clonal group (Supplementary Fig. S1 in JMM Online). All MRSA gel patterns were identical to that of the local dominant ST239 clone (Hsu et al., 2005). The majority of subjects with repeat hospitalizations were colonized by CoNS with PFGE profiles that were identical to their initial results (Supplementary Fig. S1).

Our study demonstrated high rates of highlevel mupirocin resistance among staphylococci isolated from patients subjected to daily chlorhexidine and mupirocin prophylaxis. Although chlorhexidine susceptibility was not formally tested, the high prevalence of qacA/B carriage and the isolation of staphylococci from patients who had daily chlorhexidine scrubs suggest that reduced susceptibility to chlorhexidine was common. PFGE data 
Table 1. Distribution of mupirocin- and antiseptic-resistance genes by mupirocin MIC and organism type

\begin{tabular}{|c|c|c|c|c|}
\hline Organism (no. of isolates)* & No. of patients $\dagger$ & Positive ileS-2 (\%) & Positive $s m r(\%)$ & Positive $q a c A / B(\%)$ \\
\hline \multicolumn{5}{|c|}{ High-level mupirocin resistance (MIC $\geqslant 512 \mu \mathrm{g} \mathrm{ml}^{-1}$ ) } \\
\hline Meticillin-resistant CoNS (66) & 19 & $66(100)$ & $28(42.4)$ & $52(78.8)$ \\
\hline Meticillin-sensitive CoNS (11) & 9 & $11(100)$ & $1(11.1)$ & $6(66.7)$ \\
\hline MRSA (6) & 3 & $6(100)$ & $2(16.7)$ & $6(100)$ \\
\hline MSSA (1) & 1 & $1(100)$ & $0(0)$ & $0(0)$ \\
\hline \multicolumn{5}{|c|}{ Low-level mupirocin resistance (MIC $8-256 \mu \mathrm{g} \mathrm{ml}^{-1}$ ) } \\
\hline Meticillin-resistant CoNS (10) & 6 & $0(0)$ & $0(0)$ & $10(100)$ \\
\hline Meticillin-sensitive CoNS (1) & 1 & $0(0)$ & $0(0)$ & $1(100)$ \\
\hline \multicolumn{5}{|c|}{ Mupirocin-susceptible $\left(\mathrm{MIC}<8 \mu \mathrm{g} \mathrm{ml}^{-1}\right.$ ) } \\
\hline Meticillin-resistant CoNS (9) & 7 & $0(0)$ & $4(44.4)$ & $6(66.7)$ \\
\hline Meticillin-sensitive CoNS (25) & 15 & $0(0)$ & $2(8.0)$ & $15(60.0)$ \\
\hline MRSA (5) & 2 & $0(0)$ & $1(20.0)$ & $3(60.0)$ \\
\hline MSSA (6) & 5 & $0(0)$ & $0(0)$ & $3(50.0)$ \\
\hline
\end{tabular}

${ }^{*}$ CoNS, coagulase-negative staphylococci.

$\dagger$ Patients may have both mupirocin-resistant and non-resistant staphylococcal isolates (both S. aureus and coagulase-negative staphylococci) cultured over time.

(Supplementary Fig. S1) suggested that this resistance is not due to the spread of a particular CoNS clone, but rather results from transmission of mobile genetic elements such as plasmids on which ileS-2 and $q a c A / B$ may be found (Pérez-Roth et al., 2001; Noguchi et al., 2005).

Some limitations of this work include the relatively small number of patients and short duration of study. The initial prevalence of high-level mupirocin resistance and qacA/B carriage among staphylococcal isolates prior to the practice change is unknown. However, given the absence of mupirocin-resistant isolates in newly admitted patients, it is likely that the baseline prevalence was low.

In conclusion, the practice of daily chlorhexidine bodywash and mupirocin prophylaxis increases the prevalence of chlorhexidine- and mupirocin-resistance genes in staphylococci amongst haemodialysis patients using catheters. This limited study suggests that the benefit, if any, of prophylactic antiseptics in patients with long-term haemodialysis catheters may only be transient, and continued surveillance for risk-benefit assessments is necessary.

\section{Acknowledgements}

We would like to thank Ms Lan Huay Ong and other members of the SGH Microbiology Laboratory for their assistance with this project. This study was funded by a grant from the Venerable Yen Pei-NKF Research Fund in 2008. All authors declare that there are no potential conflicts of interest.

\section{Boon Wee Teo, ${ }^{1}$ Selwyn Jun-Xi Low, ${ }^{1}$ Ying Ding, ${ }^{1}$ Tse Hsien Koh ${ }^{2}$ and Li-Yang $\mathrm{Hsu}^{1}$}

\section{${ }^{1}$ National University Health System, Singapore \\ ${ }^{2}$ Singapore General Hospital, Singapore \\ Correspondence: Li-Yang Hsu \\ (liyang_hsu@yahoo.com)}

Batra, R., Cooper, B. S., Whiteley, C., Patel, A. K., Wyncoll, D. \& Edgeworth, J. D. (2010). Efficacy and limitation of a chlorhexidine-based decolonization strategy in preventing transmission of methicillin-resistant Staphylococcus aureus in an intensive care unit. Clin Infect Dis 50, 210-217.

Bleasdale, S. C., Trick, W. E., Gonzalez, I. M., Lyles, R. D., Hayden, M. K. \& Weinstein, R. A. (2007). Effectiveness of chlorhexidine bathing to reduce catheter-associated bloodstream infections in medical intensive care unit patients. Arch Intern Med 167, 2073 2079.

Hsu, L. Y., Koh, T. H., Singh, K., Kang, M. L., Kurup, A. \& Tan, B. H. (2005). Dissemination of multisusceptible methicillin-resistant Staphylococcus aureus in Singapore. J Clin Microbiol 43, 2923-2925.

Maslow, J., Slutsky, A. \& Arbeit, R. (1993). The application of pulsed-field gel electrophoresis to molecular epidemiology. In Diagnostic Molecular Microbiology: Principles and
Applications, pp. 563-572. Edited by H. Persing, T. Smith, F. Tenover \& T. White. Washington, DC: American Society for Microbiology.

McCann, M. \& Moore, Z. E. (2010). Interventions for preventing infectious complications in haemodialysis patients with central venous catheters. Cochrane Database Syst $\operatorname{Rev}(1), \mathrm{CD} 006894$.

Noguchi, N., Suwa, J., Narui, K., Sasatsu, M., Ito, T., Hiramatsu, K. \& Song, J.-H. (2005).

Susceptibilities to antiseptic agents and distribution of antiseptic-resistance genes $q a c A / B$ and $s m r$ of methicillin-resistant Staphylococcus aureus isolated in Asia during 1998 and 1999. J Med Microbiol 54, 557565.

Pérez-Roth, E., Claverie-Martín, F., Villar, J. \& Méndez-Alvarez, S. (2001). Multiplex PCR for simultaneous identification of Staphylococcus aureus and detection of methicillin and mupirocin resistance. J Clin Microbiol 39, 40374041.

Sheng, W. H., Wang, J. T., Lauderdale, T. L., Weng, C. M., Chen, D. \& Chang, S. C. (2009). Epidemiology and susceptibilities of methicillin-resistant Staphylococcus aureus in Taiwan: emphasis on chlorhexidine susceptibility. Diagn Microbiol Infect Dis 63, 309-313.

Tacconelli, E., Carmeli, Y., Aizer, A., Ferreira, G., Foreman, M. G. \& D'Agata, E. M. (2003).

Mupirocin prophylaxis to prevent Staphylococcus aureus infection in patients undergoing dialysis: a meta-analysis. Clin Infect Dis 37, 1629-1638.

Teo, B. W., Ma, V., Xu, H., Li, J., Lee, E. J. \& Nephrology Clinical Research Group (2010). Profile of hospitalisation and death in the first 
year after diagnosis of end-stage renal disease in a multi-ethnic Asian population. Ann Acad Med Singapore 39, 79-87.
Teo, B. W., Sau, P. Y., Xu, H., Ma, V., Vathsala, A. \& Lee, E. J. (2011). Microbiology of tunnelled catheter-related infections in a multi-ethnic South-East Asian patient population. Nephron Clin Pract 118, c86-c92. 